\title{
Scoping Review on Use of Drugs Targeting Interleukin 1 Pathway in DIRA and DITRA
}

Francisco Gómez-García • Juan L. Sanz-Cabanillas • Isabel Viguera-Guerra •

Beatriz Isla-Tejera · Antonio Vélez-García Nieto · Juan Ruano (i)

Received: September 26, 2018 / Published online: November 3, 2018

(C) The Author(s) 2018

\section{ABSTRACT}

Introduction: Deficiencies in interleukin (IL)-1 receptor (IL-R) antagonist (DIRA) and IL-36R antagonist (DITRA) are rare genetic autoinflammatory diseases related to alterations in

Enhanced Digital Features To view enhanced digital features for this article go to https://doi.org/10.6084/ m9.figshare.7258685.

Electronic supplementary material The online version of this article (https://doi.org/10.1007/s13555018-0269-7) contains supplementary material, which is available to authorized users.

F. Gómez-García · J. L. Sanz-Cabanillas .

I. Viguera-Guerra · B. Isla-Tejera · A. V.-G. Nieto ·

J. Ruano $(\bowtie)$

Immune-Mediated Inflammatory Skin Disease

Research Group, IMIBIC/Reina Sofía University

Hospital/University of Córdoba, Avenida Menéndez

Pidal, Córdoba 14004, Spain

e-mail: juanruanoruiz@mac.com

F. Gómez-García · J. L. Sanz-Cabanillas .

A. V.-G. Nieto · J. Ruano

Department of Dermatology, Reina Sofía University Hospital, Avenida Menéndez Pidal, Córdoba 14004, Spain

I. Viguera-Guerra

Agencia de Evaluación de Tecnologías Sanitarias de Andalucía (AETSA), Consejería de Salud, Avda. de la Innovación, Seville 41020, Spain

B. Isla-Tejera

Department of Pharmacy, Reina Sofía University Hospital, Avenida Menéndez Pidal, Córdoba 14004, Spain antagonists of the IL-1 pathway. IL-1 antagonists may represent therapeutic alternatives. Here, we aim to provide a scoping review of knowledge on use of IL-1-targeting drugs in DIRA and DITRA.

Methods: An a priori protocol was published, and the study was conducted using the methodology described in the Joanna Briggs Institute Reviewer's Manual and the recently published PRISMA Extension for Scoping Review statement. A three-step search using MEDLINE and EMBASE databases until March 2018 with additional hand searching was performed. Data charting was performed. The search, article selection, and data extraction were carried out by two researchers independently.

Results: Twenty-four studies on use of anti-IL-1 drugs were included [15 studies including patients with diagnosis of DIRA $(n=19)$ and 9 studies including patients with diagnosis of DITRA $(n=9)]$. Most studies followed a multicenter observational design. Among all patients who received treatment with anti-IL-1 drugs, nine and four mutations in ILIRN and IL36RN were found, respectively. Patients with DIRA were treated with anakinra $(n=17)$, canakinumab $(n=2)$, or rinolacept $(n=6)$. All patients with DITRA were treated with anakinra, and only one case was also treated with canakinumab. Time-to-response frequencies were evaluated as immediate, short, and medium-long term for DIRA $(17 / 17,15 / 17$, and 9/10, 
respectively) and DITRA $(7 / 9,3 / 9$, and 2/9, respectively). Most DITRA patients in whom anti-IL-1 treatment failed experienced good response to anti-tumor necrosis factor alpha or anti-IL-12/23 drugs. The safety profiles of treatments were similar in both diseases.

Conclusions: Evidence on use of anti-IL-1 drugs in DIRA and DITRA is scarce and based on observational studies. Larger studies with better methodological quality are needed to increase confidence in use of these drugs in patients with DIRA and DITRA.

Keywords: Anakinra; Anti-IL-1 drugs; Canakinumab; Deficiency of interleukin(IL)-1 receptor(R) antagonist (DIRA); Genetic autoinflammatory diseases; Deficiency of IL36R antagonist (DITRA); Rilonacept; Scoping review

\section{INTRODUCTION}

Hereditary autoinflammatory diseases are a heterogeneous group of rare genetic disorders in which an exaggerated innate immune response is the pathogenic basis of recurrent episodes of inflammation. These inflammatory episodes affect the skin, joints, bones, eyes, gastrointestinal tract, and central nervous system in association with nonspecific signs of systemic inflammation and a lack of high-titer circulating auto-antibodies or antigen-specific $\mathrm{T}$ cells [1].

Alterations in the interleukin (IL)-1 pathway have been shown to be involved in the pathogenesis of some diseases. IL-1 contributes to coordination of the early response of the immune system to exogenous and endogenous antigens, serving as a prototypic alarm cytokine [2]. The interleukin IL-1 family comprises 11 members, including seven proinflammatory agonists (IL- $1 \alpha$, IL-1 $\beta$, IL-18, IL-33, IL-36 $\alpha$, IL-36 $\beta$, and IL-36 $\gamma$ ) and four defined or putative antagonists [IL-1 receptor (IL-1R) antagonist (IL-1RA), IL-36R antagonist (IL36RA), IL-37, and IL-38], which exert antiinflammatory activity [3]. IL-1-related hereditary diseases are considered secondary to genetic defects in both activating and inhibitory pathway molecules. Notably, cryopyrin-associated autoinflammatory syndromes (CAPSs) are primary diseases caused by alterations in IL- 1 pathway agonists. Moreover, several new diseases associated with alterations in IL-1RA, such as deficiency of IL-1RA (DIRA) and deficiency of IL36RA (DITRA), have recently been identified. IL1RA, encoded by the IL1RN gene, functions as a decoy protein that binds to IL-1R but does not result in any response signal. IL-36RA, encoded by the IL36RN gene, has been shown to specifically inhibit IL-1-induced activation of nuclear factor$\kappa B$. Both IL-1RA and IL-36RA are encoded on chromosome 2 and show 44\% homology. However, IL-1RA is expressed ubiquitously, whereas IL-36R expression is restricted to epithelial cells, including those in the skin [4]. This fact may explain the observation that both DIRA and DITRA share similar skin manifestations but with the difference that extracutaneous involvement is observed in DIRA.

DIRA is a rare life-threatening autoinflammatory disease first described in 2009 [1, 5]. DIRA is caused by autosomal recessive mutations in the $I L 1 R N$ gene and presents clinically as early onset of generalized cutaneous pustulosis, multifocal osteomyelitis, and high levels of acute-phase reactants. DITRA is a more recently described hereditary autoinflammatory disease caused by homozygous or compound heterozygous mutations in the IL36RN gene and characterized by repeated flares of generalized pustular psoriasis associated with high fever, asthenia, and systemic inflammation [6].

Various treatment strategies, such as antiinflammatories [nonsteroidal antiinflammatory drugs (NSAIDs) and corticosteroids], antimicrobial agents (antifungal and antibacterial agents), and immunosuppressants (methotrexate, cyclosporine, inhibitors of tumor necrosis factor- $\alpha$, and anti-IL-17 monoclonal antibodies), have been applied for treatment of DIRA and DITRA, with variable responses. As knowledge of the pathogenesis of these diseases has improved, increasingly specific treatments have been developed. Accordingly, four drugs targeting the IL-1 pathway have been developed. These include anakinra, a recombinant IL-1RA that competes with IL-1R agonists for its receptor [7]; rilonacept, which acts as a soluble decoy to prevent activation of IL-1RI [8]; 
canakinumab, a human monoclonal antibody targeting IL-1 $\beta$ [9]; and MABp1, an anti-IL-1 $\alpha$ monoclonal antibody [10]. Although the specificities of their mechanisms of action suggest the feasibility of achieving sufficient efficacy, the molecular differences found in both DIRA and DITRA can yield different levels of response. Moreover, these drugs are associated with short- and long-term adverse effects. Therefore, it is necessary to consider treatments with the best risk-benefit balance.

However, because DIRA and DITRA are rare diseases that have only been identified within the last decade, available evidence on use of IL-1 pathway-modulating agents for their treatment is scarce, and no secondary research has been published to date. Therefore, it is necessary to synthesize evidence derived from primary studies on use of anti-IL-1 drugs for treatment of DIRA and DITRA, map the published articles, and study the epidemiology of genetic characteristics and anti-IL-1 drug efficacy/safety results based on available evidence. Such analysis will help identify gaps in knowledge and formulate questions that can be answered through systematic review.

A scoping review is a form of scientific synthesis that addresses an exploratory research question, aimed at mapping key concepts and identifying research gaps related to a defined area or field by systematically searching, selecting, and synthesizing existing knowledge [11]. Scoping reviews contribute to integrating optimal research evidence available with clinical experience and patient values to improve care, health, and cost outcomes [12].

Accordingly, in this work, we present the results of a scoping review on use of anti-IL-1 drugs in dermatological diseases whose protocols have been previously published [13]. In this first summary, we report and discuss evidence regarding use of these drugs in patients with DIRA/DITRA.

\section{METHODS}

A scoping review protocol was a priori published [13]. The study was conducted and reported using the methodology described in the Joanna Briggs Institute Reviewer's Manual [14] and the PRISMA Extension for Scoping Reviews [15]. When necessary, authors of articles were contacted to obtain more data or clarify issues.

\section{Eligibility Criteria}

To be included in the review, papers had to show evidence of use of anti-IL-1 drugs (anakinra, canakinumab, rilonacept, or AMB1) in patients with DIRA or DITRA. Studies were included if they were written in English, involved human participants, and described the conditions formulated in the research question, regardless of publication date or format. Articles were excluded if they did not fit into the conceptual framework of the study (i.e., if a case of DIRA or DITRA was described as not having been treated with anti-IL-1 drugs). Nonscientific reviews and opinion articles were excluded.

\section{Literature Search}

We performed a three-step literature search to identify and locate all relevant studies on use of anti-IL-1 drugs in dermatological diseases, and subsequently selected articles related to use of these drugs in patients with DIRA or DITRA. The first step was performed on 9 March 2018 and entailed a limited search strategy [SEARCH QUERY ('skin disease' AND (('interleukin 1beta'/exp OR 'interleukin 1beta') OR 'interleukin 1')) AND 'therapy'] in MEDLINE and EMBASE databases. Next, we extracted keywords from titles, abstracts, and indexing categorizations and used them to perform a new search on 10 March 2018 with a more complex strategy (Table S1 in Supplementary Information). Results were ordered by disease based on review of the title and abstract. Subsequently, sources related to DIRA and DITRA were selected. The third step involved a new search using the reference lists of all selected reports and articles for identification of additional relevant studies. The literature search and title and abstract filtering were carried out independently by two researchers (F.G.-G. and J.L.S.-C.). 


\section{Data Charting}

A data charting form was jointly developed by two reviewers to determine which variables to extract. A pilot test was carried out with three studies, and the chosen variables were included in a .csv file. The two reviewers independently charted the data, discussed the results, and continuously updated the data charting form in an iterative process. Finally, variables related to patients (disease, drug, treatment response, and safety profile) and to the study design and metadata from primary sources were reported (Tables 1, 2, 3, and 4; Tables S1-S4 in Supplementary Files).

\section{Collating, Summarizing, and Reporting Results}

We grouped the articles by disease type. The results of the comprehensive search were presented using a PRISMA flow diagram. A narrative and qualitative synthesis of DIRA and DITRA mapping studies and epidemiological, genetics, and efficacy and safety findings were elaborated using tables and figures.

\section{Compliance with Ethics Guidelines}

This article is based on previously conducted studies and does not contain any studies with human participants or animals performed by any of the authors.

\section{Protocol versus Overview}

Our planned search strategy published in Dermatology and Therapy was compared with the final reported review methods. Due to time and funding limitations, we decided to select only studies published in English.

\section{RESULTS}

\section{Search Results}

From 3708 articles regarding use of anti-IL-1-related drugs in dermatological diseases, after filtering duplicates and selecting studies according to title, abstract, and keywords, 77 studies met the criteria for full-text review. Of these, 21 [DIRA $(n=13)$ and DITRA $(n=8)]$ fulfilled the inclusion criteria. After reviewing citations of references for these studies, three new articles [DIRA $(n=2)$ and DITRA $(n=1)]$ were obtained. Finally, 24 studies, including 15 describing DIRA $(n=15)$ and 9 describing DITRA $(n=9)$, were included in the scoping review. A reference list of all included and excluded articles with reasons for exclusion is presented in Fig. S1 in Supplementary Information.

\section{Evidence for Mapping the Studies of IL-1- Based Treatment in Patients with DIRA/ DITRA}

\section{DIRA}

Fifteen studies, published between 2009 and 2017 as full papers $(n=11)$ or congress abstracts $(n=4)$, followed mainly an observational design (13 case reports, 1 case series), and only one was an intervention study. Only three studies (20\%) had an a priori protocol that was included in a public registry. Twelve (63.1\%) were elaborated as multicentric studies involving up to nine different institutions. The average numbers of authors and affiliations per article were 11 (range 3-45) and 5 (range 2-14), respectively, and different medical specialties were involved [pediatrics $(n=11)$, genetics $(n=11)$, rheumatology $(n=7)$, allergy-immunology $(n=6)$, and dermatology $(n=5)]$, although authors from academia $(n=4)$ and the pharmaceutical industry $(n=1)$ were also included. Most studies were performed in the USA [8], with the National Institutes of Health (NIH, Bethesda, MD, USA) and associated centers of institutions participating in more studies $(n=10$; Table S1 in Supplementary Information). Other countries that also participated were Brazil $(n=3)$, Turkey $(n=2)$, Germany $(n=1)$, and Puerto Rico $(n=1)$. The studies were published in journals from disciplines such as rheumatology $(n=5)$, general medicine $(n=4), \quad$ dermatology $(n=3)$, immunology $(n=2)$, and radiology $(n=1)$.

In seven $(46.7 \%)$ and ten $(66.7 \%)$ studies, disclosures related to the source of funding 


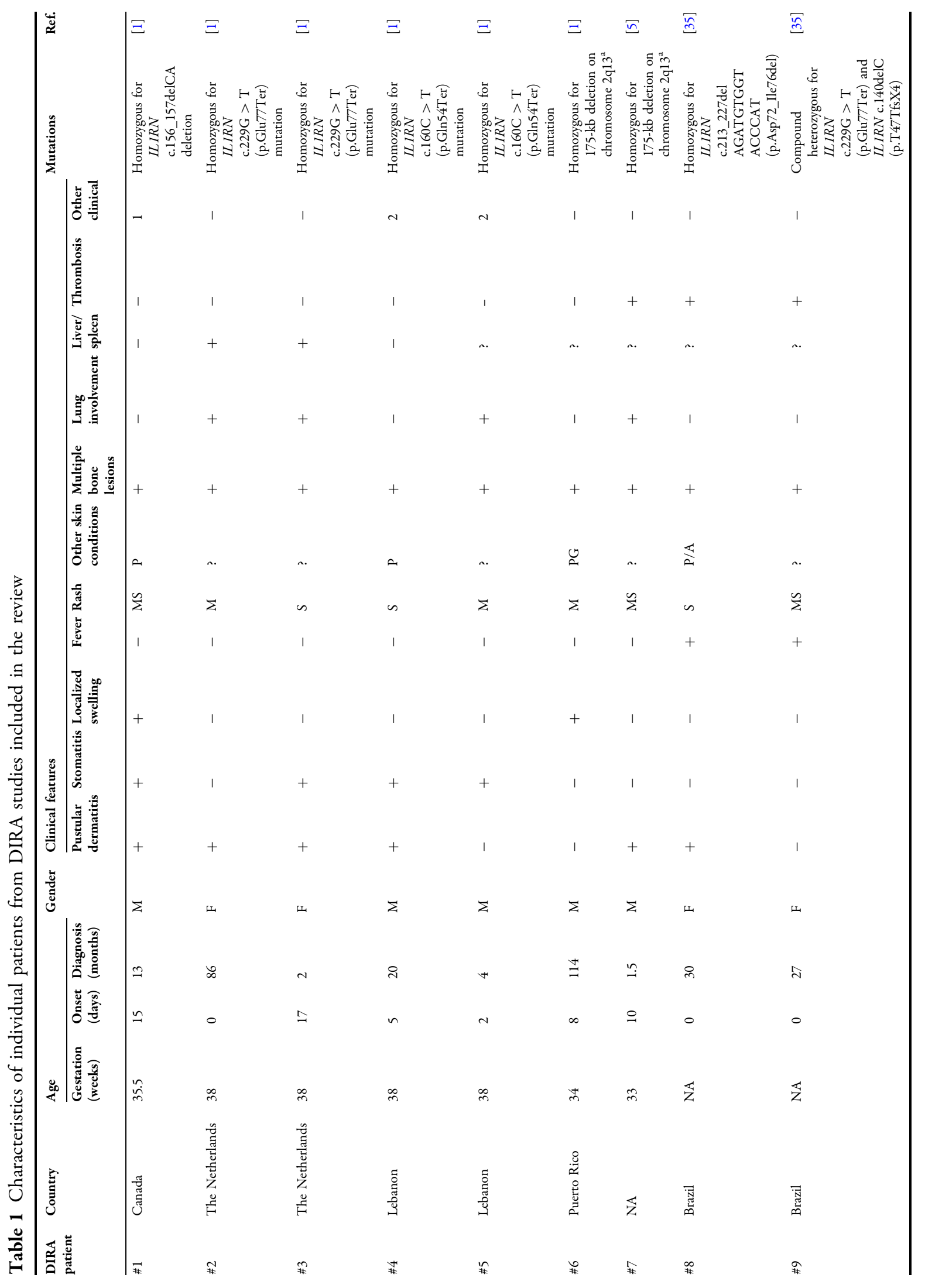




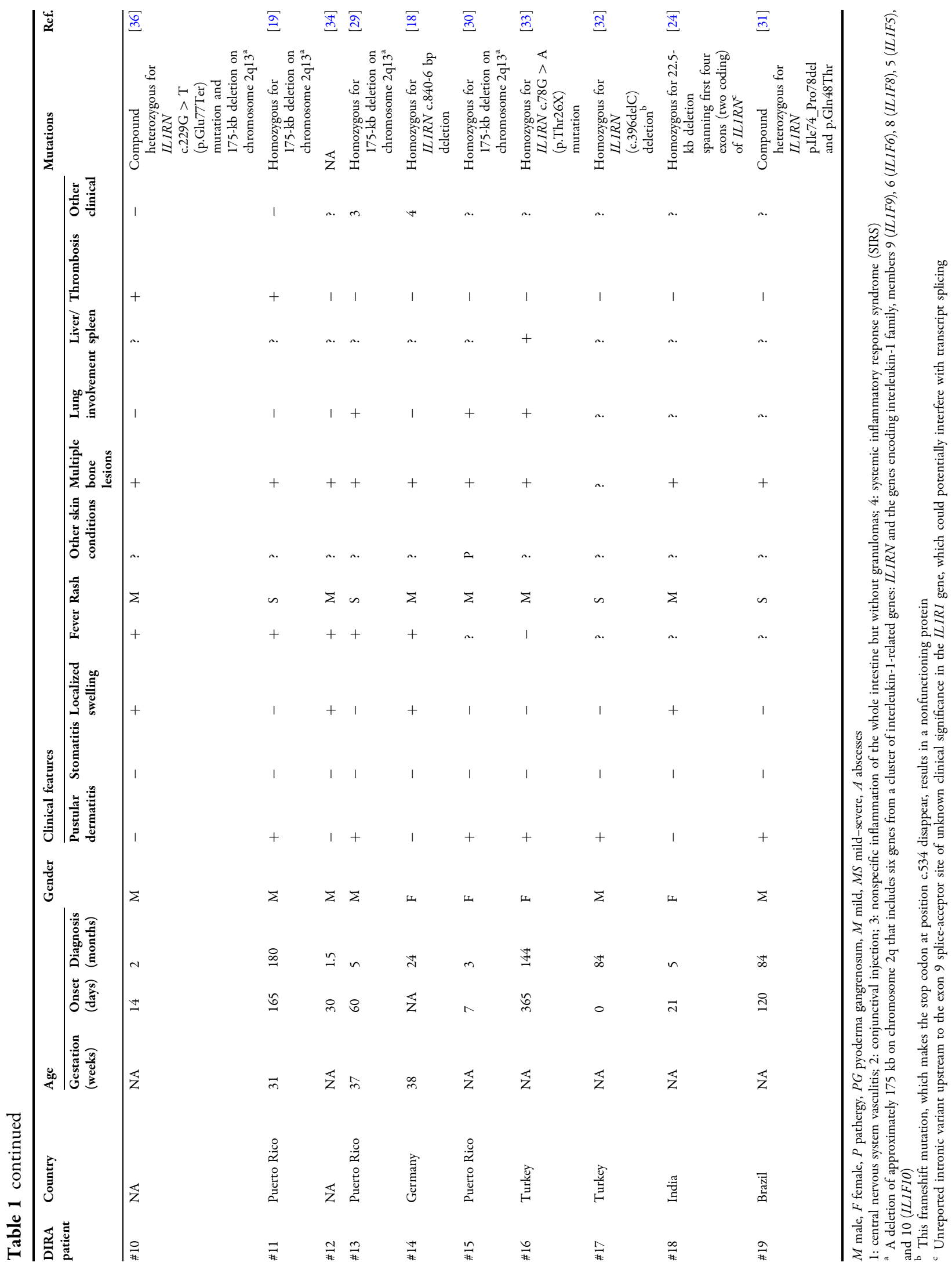




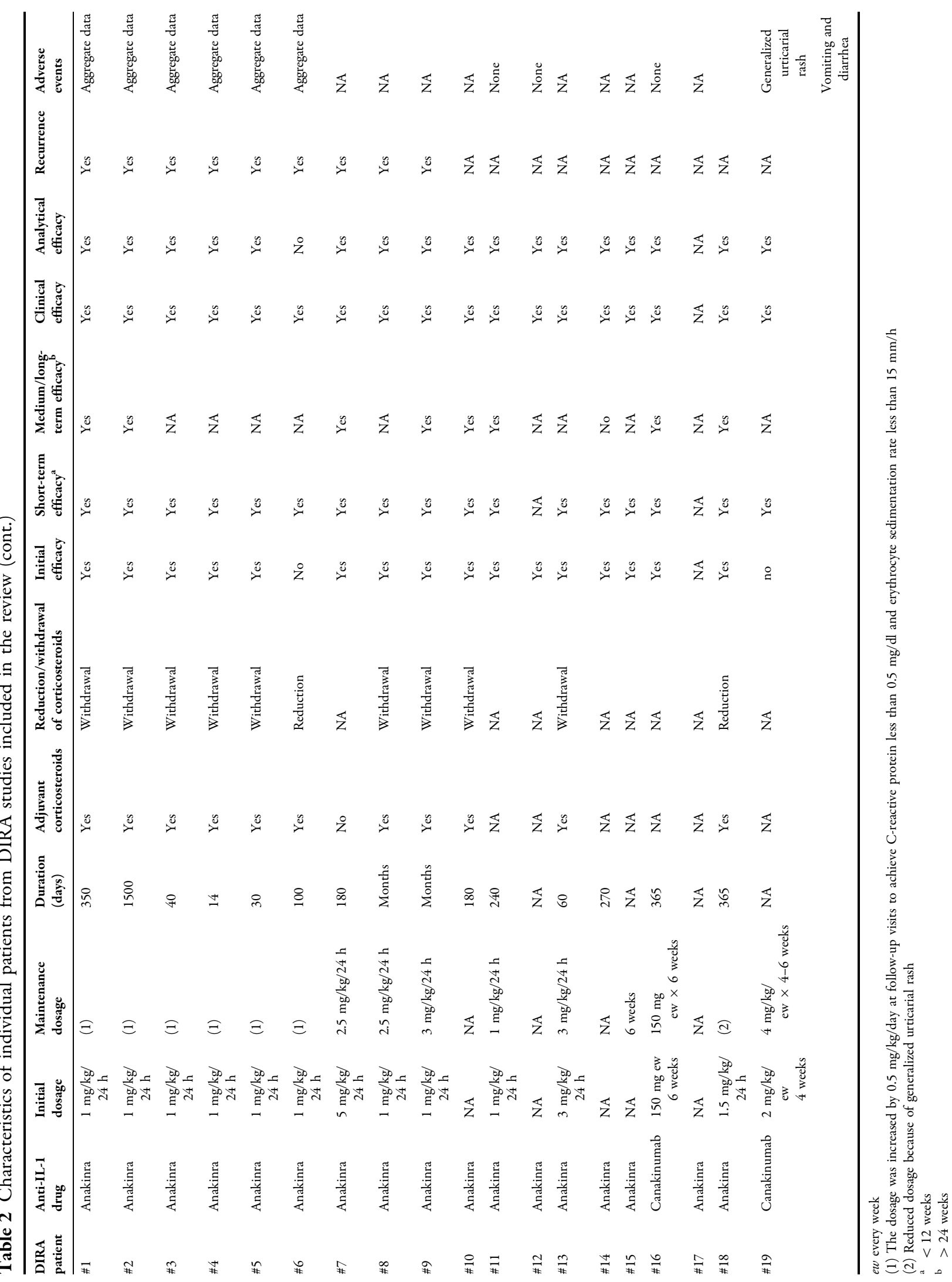




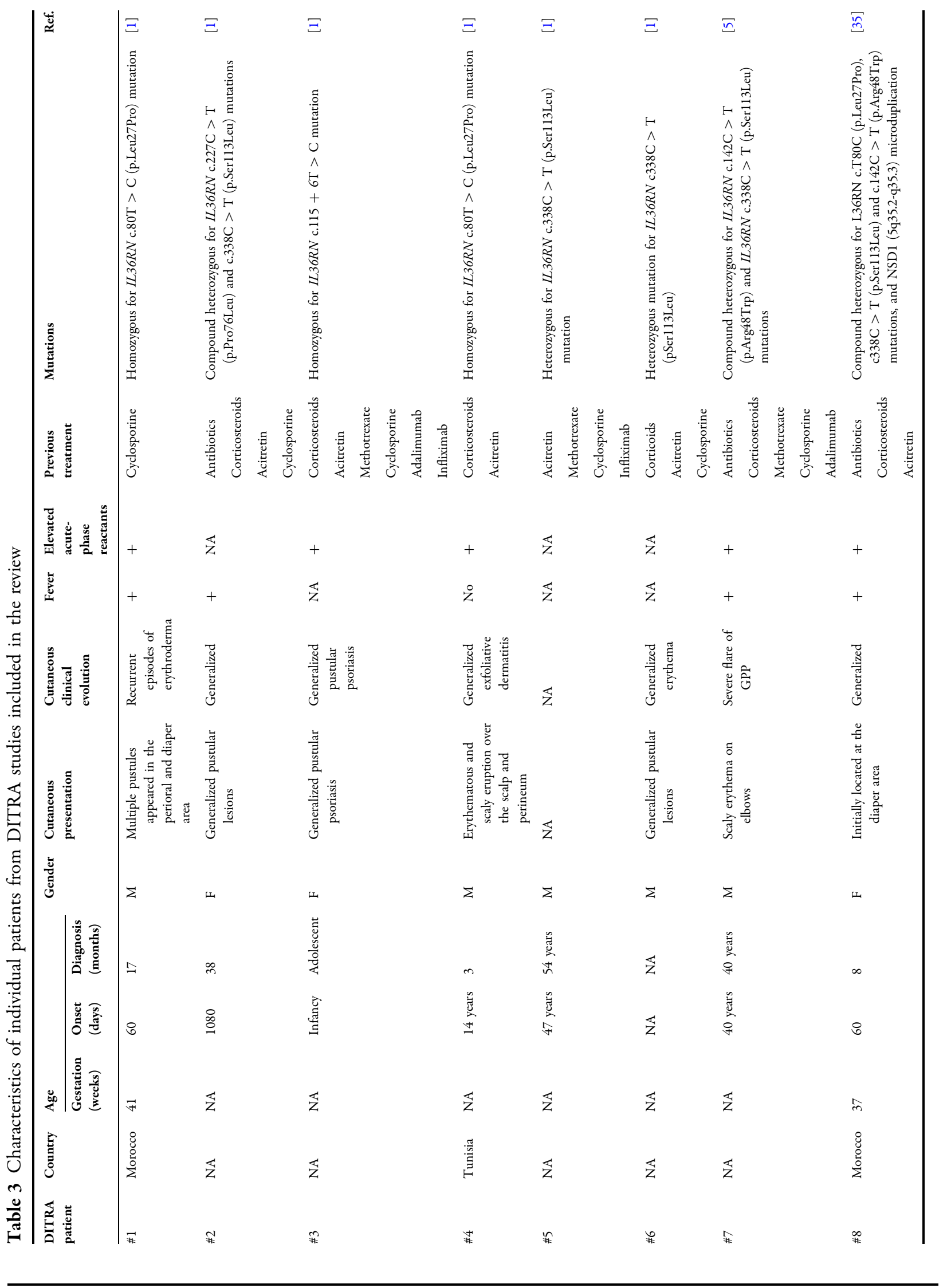




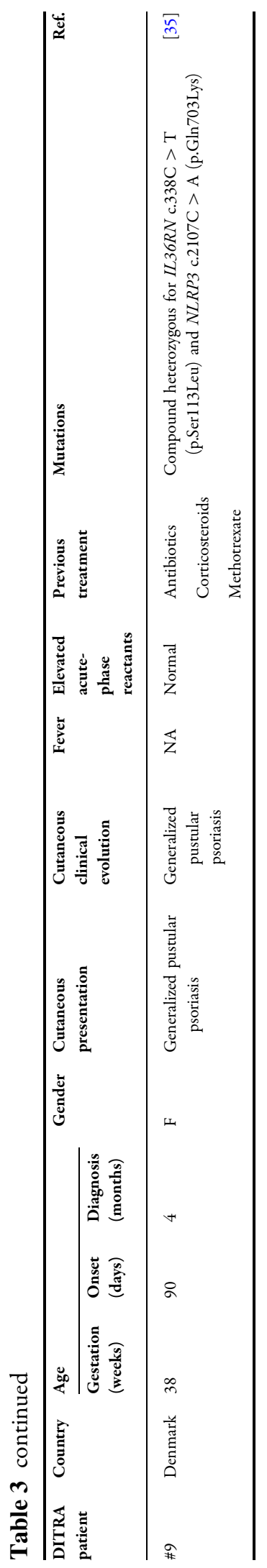

(five, public sources; one, public and pharmaceutical sources; and one without funding) or authors' conflicts of interest (CoIs) were declared, respectively. AMGEN (five authors), Novartis (two authors), and Lilly (two authors) were the pharmaceutical companies most frequently cited in CoIs by the authors.

\section{DITRA}

Nine studies related to use of anti-IL-1 drugs in DITRA were found. All were observational case report studies. No a priori design or registration was reported for any of these studies. Five of nine $(55.5 \%)$ were multicenter studies (range 1-5). No centers participated in more than one study. Two were performed in Spain and France, and one each was performed in The Netherlands, USA, Italy, Denmark, and Germany. Articles were published between 2011 and 2018. Six papers were published in dermatology journals, two in rheumatology journals, and one in a pediatrics journal. Three were full papers, three were letters, and three were abstracts from conferences. The average numbers of authors and affiliations per article were 7.2 (range 4-15) and 3.22 (range 1-7), respectively. The most frequent specialties were dermatology in eight papers and genetics and pediatrics in five papers. One study [16] declared funds from Strasbourg High Throughput Next Generation Sequencing facility (GENOMAX) and INSERM UMR_S 1109. Four did not report funding or did not provide funding information. In two of the studies, the authors declared CoIs (Novartis and Sobi for three and two authors, respectively; these companies were the most frequently declared in CoIs). Three and four papers declared no CoIs or did not provide such information, respectively.

\section{Evidence for Analysis of Epidemiology of IL-1-Based Agents in Treatment of Patients with DIRA/DITRA}

\section{DIRA}

Individual data were obtained from 19 patients who had been treated with anakinra $(n=17)$ and canakinumab $(n=2)$. Eight of $19(42.10 \%)$ patients were female. Of these 19 patients, 3 


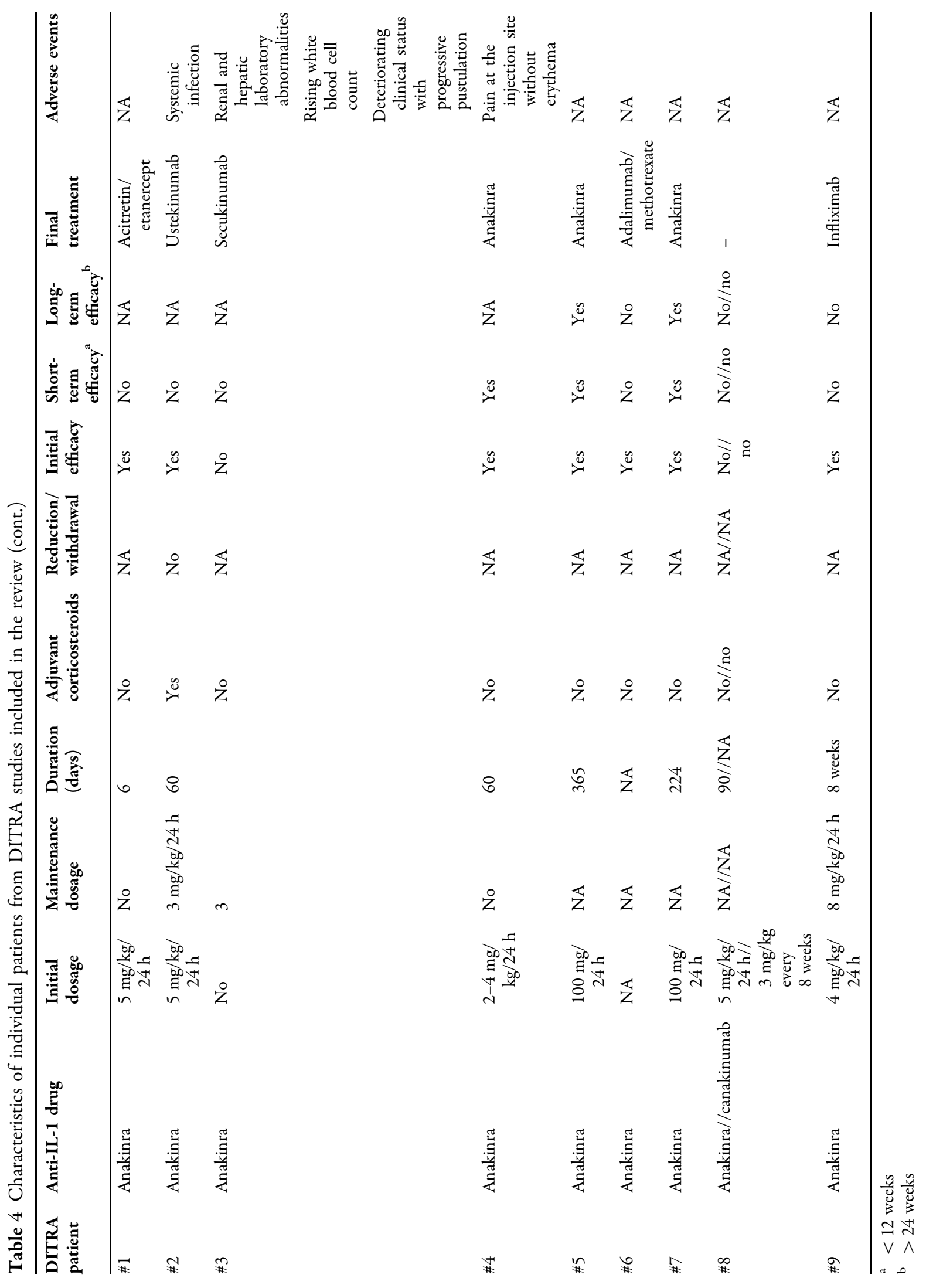


each were from Brazil and Puerto Rico, 2 each were from Turkey, Lebanon, and The Netherlands; and 1 each was from India, Canada, and Germany. Data from three patients could not be obtained. Seven of $11(63.63 \%)$ patients were full-term infants, and 4 of $11(36.36 \%)$ were preterm newborns. Data for eight patients were missing. Pustular dermatitis and localized swelling were observed in 12 and 6 patients, representing the most frequent symptom of onset of the disease. During disease evolution, patients presented with pustular skin rash (severe in 6 of 19 cases), multiple bone involvement (ribs being the most frequent site, in 17 of 19), fever (in 8 of 16), lung involvement (in 7 of 19), venous thrombosis (in 5 of 19), and elevation of acute-phase reactants (in 19 of 19). With respect to age at disease presentation, data were obtained from 18 patients. The median age was 14 days (range 0-365 days). Median age at diagnosis was 24 months (range 1.5-180 months). The median delay in diagnosis was 19.5 months (range $0.5-179.5$ months). Seventeen of 18 (94.4\%) patients had received previous treatment. Of these, 13 were treated with corticosteroids, 12 with antibiotics, 4 with NSAIDs, 3 with antifungals, 3 with acitretin, and 1 each with methotrexate, cyclosporine, intravenous immunoglobulins, azathioprine, thalidomide, and etanercept.

One study [17] presented aggregate data from six patients who had been treated with rinolacept. These patients received pretreatment with anakinra and were included among the 19 previously exposed patients described above.

\section{DITRA}

Individual data were obtained from nine patients. All had been treated with anakinra, and one had also received canakinumab. Three of eight (37.5\%) were female. One patient had missing data. Two patients were from Morocco, and one patient each was from Tunisia and Denmark. The origins of the remaining patients were not reported. Onset disease was localized in four patients (two for the diaper and scalp, two for the elbow and perineum) and generalized in four patients. During disease evolution, the disease worsened, presenting with erythroderma or pustules in all patients for whom data were available. The process was accompanied by fever in four of six $(66.6 \%)$ patients and elevation of acute inflammation reactants in five of six $(83.3 \%)$ patients. The ages at onset and disease diagnosis varied from neonate to adulthood (14 days to 47 years and 3 months to 54 years, respectively). The delay in diagnosis ranged from 2 months to 17 years. All patients previously received other treatments, including corticosteroids in seven patients, acitretin and ciclosporin in six patients each, methotrexate in four patients, cyclosporine in three patients, and adalimumab and infliximab in two patients each.

\section{Evidence of Different Genotypic Variations in Patients with DIRA/DITRA Treated with IL-1 Inhibitors}

\section{DIRA}

Nine different mutations in IL1RN, showing a homozygous genotype in all cases, were described among patients with DIRA treated with anti-IL-1 drugs in the included studies. A genomic $175-\mathrm{kb}$ deletion on chromosome 2 was observed in patients \#6, \#7, \#11, \#13, and \#15; two nonsense mutations were detected in patients \#2, \#3, and \#10 (c.229G $\rightarrow \mathrm{T}$ ) and patients \#4 and \#5 (c.160C $\rightarrow$ T); a 15-bp frame deletion (c. 213_227delAGATGTGGTACCCAT resulting in p.Asp72_Ile76del) was observed in patients \#8 and \#9; 2-bp (c.156_157delCA) and $22-\mathrm{kb}^{1}$ frameshift deletions were identified in patients \#1 and \#18, respectively; and an intronic variant of unknown clinical significance in the IL1R1 gene (position c.840, 6 bp upstream of the exon 9 splice-acceptor site), which could potentially interfere with transcript splicing machinery, was found in patient \#14. Additionally, the p.R26X IL1RN mutation was observed in patient \#16, c.396delC nucleotide deletion was observed in patient \#17, and both p.Ile74_Pro78del and p.Gln48Thr

\footnotetext{
1 This frameshift spans four exons (two coding) of $I L 1 R N, Q 54 X$, and an unreported intronic variant of unknown clinical significance in the IL1R1 gene. This variant resides at position c.840, $6 \mathrm{bp}$ upstream of the exon 9 splice-acceptor site and could potentially interfere with transcript splicing.
} 
IL1RN mutations were observed in patient \#19. No mutation analysis was performed in patient $\# 12$.

\section{DITRA}

Thee different homozygous and heterozygous mutations in IL36RN were described among patients with DITRA treated with anti-IL-1 drugs in the included studies. IL36RN c.80T $>\mathrm{C}$ (p.Leu27Pro) was the most frequent homozygous mutation, present in patients \#1, \#4, and \#8. Patient \#8 also presented a microduplication including the NSD1 gene (5q35.2-q35.3). Homozygous mutation at c. $115+6 \mathrm{~T}>\mathrm{C}$ in patient \#3 was also reported. Patient \#6 was diagnosed with an homozygous mutation for IL36RN, but the position was not reported. Regarding heterozygous mutations, patients \#2, $\# 6$, and \#8 were identified as carriers of IL36RN c338C $>$ T (pSer113Leu) at exon 5, in patients $\# 2$, \#6, \#8, and \#9. Patients \#8 and \#9 also presented a heterozygous mutation in IL36RN c.142C $>\mathrm{T}$ (p.Arg48Trp) and NLRP3 c.2107C $>$ A (p.Gln703Lys) at exon 3, respectively.

\section{Evidence for Efficacy and Safety of IL-1- Based Agents in Treatment of DIRA/DITRA}

\section{DIRA}

We obtained individual data for 17 patients treated with anakinra and 2 patients treated with canakinumab. Length of therapy varied between 2 weeks and 4.5 years for anakinra and was up to 12 months for canakinumab. The initial dosage of anakinra ranged from 1 to $5 \mathrm{mg} / \mathrm{kg} /$ day, with $1 \mathrm{mg} / \mathrm{kg} /$ day being the dosage most frequently used, in 9 of 13 patients $(69.2 \%)$ for whom data were available. The dosage was increased in eight patients up to $2.5-3 \mathrm{mg} / \mathrm{kg} /$ day or until achieving clinical and analytical response. Two patients required dosage reduction, one due to achieving therapeutic response and the other due to development of generalized urticaria. In two other patients, the initial dosage was maintained. In 11 patients treated with anakinra, concomitant use of corticosteroids was reported; in nine of these cases, the drugs were finally withdrawn, and in two cases, dosage reduction was performed. All patients achieved immediate (day-hours) clinical responses, and all but one patient also showed an analytical response. The patient who did not show complete response to anakinra was homozygous for a deletion of approximately $175 \mathrm{~kb}$ on chromosome 2q, which harbored six genes from a cluster of IL-1related genes, i.e., IL1RN and genes encoding IL1 family members 9 (IL1F9), 6 (IL1F6), 8 (IL1F8), 5 (IL1F5), and 10 (IL1F10) [1].

In the short term ( $<12$ weeks), 15 (88.2\%) patients showed good clinical response. In two cases, response was not reported. Ninety percent of patients for whom data were available in the medium/long term ( $>24$ weeks) $(n=10)$ showed good response. The nonresponder in this group showed a clinical phenotype of chronic recurrent multifocal osteomyelitis and finally responded to azathioprine, corticosteroids, and intravenous immunoglobulins [18].

In the two patients treated with canakinumab, one showed good immediate, short-term, and medium/long-term response, and the other required dosage increase to achieve good response in the short term. No medium/longterm data were available for this patient.

With respect to drug safety, anakinra was associated with some adverse events, including transient injection-site reactions $(n=3)$ and anaphylactic reactions $(n=2)$. An episode of vomiting and diarrhea was reported in a patient treated with high dosage of canakinumab. Finally, subsequent discontinuation of anakinra in nine patients was reported following a flareup of their disease.

Aggregated data from six patients with DIRA treated with rinolacept were reported in an intervention study with follow-up visits at 3, 12, and 24 months. These patients had previously received anakinra, and their data were among those published individually, corresponding to patients \#8, \#9, \#10, \#13, and \#15. The sixth patient corresponded to one of those included in the article by Aksentijevich et al. (Table S1 of Supplementary Information). All patients were treated with an initial bolus of $4.4 \mathrm{mg} / \mathrm{kg} / \mathrm{dose}$ rilonacept and were discharged on $2.2 \mathrm{mg} / \mathrm{kg} /$ week. All except one patient required rilonacept dosage escalation to $4.4 \mathrm{mg} / \mathrm{kg} /$ week due to 
partial clinical response. All patients remained in clinical and analytical remission. Rilonacept was well tolerated, no serious adverse events were reported, and none of the patients met protocol adverse event criteria for rilonacept dosage reduction or permanent discontinuation. The most common events were upper respiratory infection (100\%), otitis media $(66.7 \%)$, and rash (66.7\%). Finally, clinical responses to rilonacept were comparable to those observed with anakinra, although there was a clear preference by relatives and patients for staying on weekly versus daily injections [17].

\section{DITRA}

Efficacy and safety data were available for nine patients with DITRA. All patients were treated with anakinra at $2-5 \mathrm{mg} / \mathrm{kg} /$ day or $100 \mathrm{mg} /$ day, and one patient was also treated with canakinumab $3 \mathrm{mg} / \mathrm{kg}$ every 8 weeks. Duration of anakinra treatment ranged from 3 days to 12 months. With regard to efficacy, $77.8 \%$ of patients who received anakinra experienced good initial (hours-days) clinical response. Of these, $33.3 \%$ maintained the response in the short term $(<12$ weeks). In the medium/long term ( $>24$ weeks), $22.2 \%$ of patients continued the treatment. One patient, in whom anakinra had previously failed, received treatment with canakinumab, and this treatment did not prove effective at the initial time or in the short- or long-term analyses [16].

With respect to the safety of anakinra, one case of systemic infection was reported, another one of renal and hepatic laboratory abnormalities, rising white blood cell count, and deteriorating clinical status with progressive pustulation and, finally, pain at the injection site without erythema. No adverse events were reported in the patient who had been treated with canakinumab.

In five of eight patients in whom anti-IL-1 drugs failed, the final therapeutic regimens were etanercept and acitretin [19], adalimumab and methotrexate [20], secukinumab [21], ustekinumab [22], and infliximab [23].

\section{DISCUSSION}

\section{Summary of Findings}

This is the first scoping review to map and summarize use of drugs targeting the IL-1 pathway in patients with DIRA and DITRA. DIRA and DITRA were first identified in 2009 and 2011, respectively, and clinical experience with use of anti-IL-1 drugs is scarce. The best available evidence is based on results from observational studies, most of which were not published in a public repository with an a priori design. Studies of both diseases have been multicenter, multidisciplinary studies, and the findings of these studies have generally been obtained from public sources, with few authors declaring CoIs.

Anakinra is the most commonly used drug for both diseases. The use of canakinumab is anecdotal, and rinolacept has only been used in DIRA. Patients with DIRA tended to be younger at time of onset and start of treatment, and a delay in diagnosis was common for patients with DITRA. No relationships were observed among initial features, evolution, or response to treatment or in the type of genetic mutations present in patients treated with anti-IL-1 drugs. The effectiveness of these treatments differs in both diseases, with greater efficacy observed in patients with DIRA than in patients with DITRA at all time points. Patients with DITRA typically showed good immediate response, but decreased short- and medium/long-term responses. No relevant differences in safety have been found.

\section{Strengths and Limitations}

Methodologically, this study was conducted based on an a priori protocol, previously published in a scientific journal and using the latest standards in scoping review methodology. In each of the phases, at least two researchers were involved. Contact with authors of some of the studies allowed us to clarify important points for analyzing the primary data. This report was made according to the recommendations of the PRISMA Extension for Scoping Reviews. 
Limitations related to funding and time prevented us from including some articles written in languages other than English. Additionally, it was not possible to contact some of the authors of the articles in order to reduce the percentage of missing data. This was particularly limiting for articles published in the form of abstracts, because we did not exclude these types of publication. This work is a substudy, and although we believe that the global search strategy was complete and that the development of the three-phase search minimized loss of relevant articles, it is possible that we have not included some papers describing studies related to the research topic. The risk of bias in the studies, the quality of the evidence, and statistical analysis techniques were not evaluated. Finally, this is the first scoping review carried out by this research team; therefore, it is possible that there may have been some other unknown limitations.

\section{Research Gaps}

The low number of studies, low quality of evidence based on study design, and lack of an a priori design included in a public repository increase the uncertainty regarding the validity of the results. The studies reported in this review were multicenter, multidisciplinary studies, and these characteristics were more evident for DIRA than for DITRA, because DIRA exhibits greater systemic involvement and is fundamentally a disease of the bone system. The included studies were performed in different countries, mostly developed countries, and this observation was more evident for DITRA than DIRA. Surprisingly, no center participated in more than one study in DITRA articles, whereas in articles on DIRA, the NIH, which included various institutions, was involved in 10 of 15 studies. Finally, the absence or public nature of the funding source and the low number of author CoIs were notable. This may be due to the small market for drugs used to treat rare diseases, which can make drugs extremely expensive. Consequently, many pharmaceutical companies may stop manufacturing these drugs or may not initiate research and development into new therapies [25]. Based on these above findings, primary studies of higher methodological quality and with a greater number of patients are necessary, and secondary scientific investigations should serve as the basis for development of clinical guidelines. Because DIRA and DITRA are rare diseases, making it difficult to include large numbers of patients and find funding, these studies should be multicenter studies, likely with a reference center (similar to that observed for DIRA), and improvement of public and private financial support is needed.

With regard to knowledge on the epidemiology and genetics of DIRA and DITRA, because the reporting of results has not been systematized, preparation of a summary of the results related to use of anti-IL-1 drugs is complex. Accordingly, it would be useful for study summaries to provide case reports for reporting protocols, such as CARE guidelines [26]. Despite this, it was possible to observe the onset of the disease and the earlier administration of treatments in patients with DIRA versus patients with DITRA. Both of these patient groups exhibited delays in diagnosis, probably due to the characteristics of rare diseases and the need for appropriate technology for genetic diagnosis, which may be particularly important in developing countries. In all of the presented cases, the existence and/or the type and position of the mutation found in ILIRN or IL36RN genes were reported. Thus, of the 43 [27] and 60 [28] homozygous or homozygous and heterozygous mutations that have been described in DIRA and DITRA, respectively, we found nine and four differences in patients who had received treatment with anti-IL-1 drugs. Given the small number of cases published, it is difficult to find associations between the form of presentation or the severity of the disease and the type of mutation in patients treated with anti-IL-1 drugs.

With regard to efficacy, we have not found validated instruments or severity criteria to evaluate the degree of improvement in patients with DIRA or DITRA following treatment. This makes it difficult to quantify the response. To date, treatment efficacy in studies of patients with DIRA has been measured by assessing 
clinical and analytical improvement. In patients with DITRA, the reported response is fundamentally clinical. Based on this limited evidence, patients with DIRA showed higher responses at all measured times and did not exhibit disease recurrence; thus, the dosage of corticosteroids can be reduced while receiving treatment with anti-IL-1 drugs. Only one patient with DIRA, who also presented with chronic recurrent multifocal osteomyelitis, showed disease recurrence when being administered anakinra [18]. However, patients with DITRA who responded well immediately showed only weak short- and medium/longterm response. Thus, these patients should be given alternative treatments. In relation to the best available evidence on use of other biological drugs in DITRA that we have been able to find, Boehner et al. recently published a systematic review on generalized pustular psoriasis, including DITRA patients [38]. Those authors found that anti-TNF (7/8) and anti-IL12/IL-23(p40) (1/1) drugs also seemed to be effective in DITRA patients. In our study, in five of eight patients in whom anti-IL-1 drugs failed, the final therapeutic regimens were etanercept and acitretin, adalimumab and methotrexate, secukinumab, ustekinumab, and infliximab. Only one patient with DITRA who had poor response to etanercept and experienced relapse after adalimumab was treated successfully with anakinra [37].

With respect to safety, data from observational studies are unreliable, and cases of paradoxical generalized pustular psoriasis, and worsening of the disease or severe infections after anti-TNF and anti-interleukin 1 treatments have been described in literature. Thus, our observations suggest that DITRA patients may experience a better response with a similar safety spectrum with anti-TNF, anti-IL-12/IL23(p40) or anti-IL-17 drugs as compared with other molecules blocking the IL-1 pathway $[39,40]$. With regard to concomitant use of corticosteroids, only one case was reported, and worsening of the disease was observed [22].

The response to canakinumab and rinolacept was generally similar to that to anakinra for both diseases in the reported cases. No differences were observed in the safety of these treatments in both diseases. Finally, no relationships between the location of the genetic mutation and the response to treatment were observed; For example, genetic alterations were present in the one patient [1] with DIRA who did not respond completely to anakinra treatment, but were also present in the other four patients (\#5, \#29, \#30, and \#31) who did respond to treatment.

\section{CONCLUSIONS}

Available evidence on use of anti-IL-1 drugs in patients with DIRA and DITRA is scarce and based on observational studies. Anakinra is the most commonly used drug in patients with DIRA or DITRA, and most experience with canakinumab has been anecdotal. Rinolacept has only been used in patients with DIRA. There was no apparent relationship between mutations found in patients treated with anti-IL-1 drugs and the onset symptoms, disease evolution, or treatment response. The observed efficacy was high in patients with DIRA at all time points and at the initial (immediate) time in patients with DITRA, but was low at short- and medium/long-term periods in these patients. No differences in safety were found.

Confidence in these results is limited by the lack of available evidence and the type of methodology used in the studies. Systematizing the collection and reporting of cases, standardizing scales of severity measurement, and having greater public/private involvement are necessary to produce primary studies that can be used to answer questions regarding the efficacy, safety, and association of mutations with response to treatments using systematic reviews and metaanalysis techniques.

\section{ACKNOWLEDGEMENTS}

We wish to acknowledge the assistance received from Dr. Gina Montealegre [Translational Autoinflammatory Disease Section (TADS), National Institute of Allergy and Infectious Diseases, NIH/NIAID/LCIM, Bethesda, US] for answering our questions about some aspects of 
the DIRA patients during the phase of the review.

Funding. This work was supported, in part, by project ICI1400136 to JR, integrated into the National Plan of R + D + I 2008-2011 and cofinanced by the ISCIII-Subdirección General de Evaluación and European Regional Development Fund (ERDF), by project PIN-0316-2017 of the Consejería de Salud, Junta de Andalucía (Spain) to JR, and by grant PP13/009 of Plan Propio de movilidad para investigadores del Instituto Maimonides de Investigacion Biomédica de Córdoba (IMIBIC). No funding was received from any pharmaceutical company. No funding was received for publication of this article.

Authorship. All named authors meet the ICMJE criteria for authorship for this manuscript, take responsibility for the integrity of the work as a whole, and have given final approval for the version to be published. Finally, the authors would like to thank Editage (www. editage.com) for English language editing.

Disclosures. Francisco Gómez-García has received honoraria for research from Pfizer, and for lecturing from Abbvie, Janssen-Cilag and Novartis; Antonio Vélez-García Nieto has received honoraria for lecturing from Pfizer, Novartis, AbbVie, and Janssen-Cilag, and other financial benefits from AbbVie, Novartis, and Janssen-Cilag; Juan Ruano has received honoraria for lecturing and grants for research from Pfizer, honoraria for lecturing from Janssen-Cilag and Novartis, and other financial benefits from Abbvie and Novartis. Juan Ruano is a member of the Cochrane Bias Methods Group and Skin Group. Francisco Gómez-García is a member of the Cochrane Bias Methods Group and Skin Group. Beatriz Isla-Tejera is a member of the Cochrane Bias Methods Group and Skin Group. Juan L. Sanz-Cabanillas and Isabel Viguera-Guerra have nothing to disclose.

Compliance with Ethics Guidelines. This article is based on previously conducted studies and does not contain any studies with human participants or animals performed by any of the authors.

Data Availability. All data generated or analyzed during this study are included in this published article/as supplementary information files.

Open Access. This article is distributed under the terms of the Creative Commons Attribution-NonCommercial 4.0 International License (http://creativecommons.org/licenses/ by-nc/4.0/), which permits any noncommercial use, distribution, and reproduction in any medium, provided you give appropriate credit to the original author(s) and the source, provide a link to the Creative Commons license, and indicate if changes were made.

\section{REFERENCES}

1. Aksentijevich I, Masters SL, Ferguson PJ, Dancey P, Frenkel J, van Royen-Kerkhoff A, et al. An autoinflammatory disease with deficiency of the interleukin-1-receptor antagonist. N Engl J Med. 2009;360:2426-37.

2. Dinarello CA. Biologic basis for interleukin-1 in disease. Blood. 1996;87:2095-147.

3. Palomo J, Dietrich D, Martin P, Palmer G, Gabay C. The interleukin (IL)-1 cytokine family-balance between agonists and antagonists in inflammatory diseases. Cytokine. 2015;76:25-37.

4. Kumar S, McDonnell PC, Lehr R, et al. Identification and initial characterization of four novel members of the interleukin-1 family. J Biol Chem. 2000;275:10308-14.

5. Reddy S, Jia S, Geoffrey R, et al. An autoinflammatory disease due to homozygous deletion of the IL1RN locus. N Engl J Med. 2009;360:2438-44.

6. Marrakchi S, Guigue P, Renshaw BR, et al. Interleukin-36-receptor antagonist deficiency and generalized pustular psoriasis. $\mathrm{N}$ Engl $\mathrm{J}$ Med. 2011;365:620-8.

7. Hallegua DS, Weisman MH. Potential therapeutic uses of interleukin 1 receptor antagonists in human diseases. Ann Rheum Dis. 2002;61:960-7. 
8. Economides AN, Carpenter LR, Rudge JS, et al. Cytokine traps: multi-component, high-affinity blockers of cytokine action. Nat Med. 2003;9:47-52.

9. Novartis Pharmaceuticals Corporation. Prescribing information for ILARIS (canakinumab). http:// www.accessdata.fda.gov/drugsatfda_docs/label/ 2012/125319s047lbl.pdf. Accessed on 31 Aug 2018.

10. Hong DS, Janku F, Naing A, et al. Xilonix, a novel true human antibody targeting the inflammatory cytokine interleukin-1 alpha, in non-small cell lung cancer. Investig New Drugs. 2015;33:621-31.

11. Colquhoun HL, Levac D, O'Brien KK, et al. Scoping reviews: time for clarity in definition, methods, and reporting. J Clin Epidemiol. 2014;67:1291-4.

12. Al-Almaie SM, Al-Baghli NA. Evidence based medicine: an overview. J Fam Community Med. 2003;10:17-24.

13. Gómez-García F, Ruano J, Gay-Mimbrera J, et al. A scoping review protocol to explore the use of interleukin-1-targeting drugs for the treatment of dermatological diseases: indications, mechanism of action, efficacy, and safety. Dermatol Ther (Heidelb). 2018;8:195-202.

14. Aromataris E, Munn Z (Editors). Joanna Briggs Institute reviewer's manual. The Joanna Briggs Institute, 2017. Available from https:// reviewersmanual.joannabriggs.org/. Accessed 18 Aug 2018.

15. Tricco AC, Lillie E, Zarin W, O'Brien $\mathrm{KK}$, et al. PRISMA extension for scoping reviews (PRISMAScR): checklist and explanation. Ann Intern Med. 2018. https://doi.org/10.7326/m18-0850 (Epub ahead of print).

16. Carapito R, Isidor B, Guerouaz N, et al. Homozygous IL36RN mutation and NSD1 duplication in a patient with severe pustular psoriasis and DITRAunrelated symptoms. Br J Dermatol. 2015;172: 302-5.

17. Garg M, de Jesus AA, Chapelle D, et al. Rilonacept maintains long-term inflammatory remission in patients with deficiency of the IL-1 receptor antagonist. JCI Insight. 2017. https://doi.org/10.1172/jci. insight.94838.

18. Zimmer C, Moll M, Rieber N, Goldbach-Mansky R, Aksentijevich I, Kuemmerle-Deschner J. IL-1 inhibition in a patient with polymorphism in the interleukin 1-receptor type 1 gene and clinical phenotype of CRMO/DIRA. Ann Rheum Dis. 2013;71(SUPPL. 3):257.

19. Cuperus W, Koevoets R, van der Smagt JJ, et al. Juvenile interleukin-36 receptor antagonist deficiency (DITRA) with c.80T $>$ C (p.Leu27Pro) mutation successfully treated with etanercept and acitretin. JAAD Case Rep. 2018;4(2):192-5.

20. Capusan TM, et al. Combined therapy in 2 cases of pustular generalized psoriasis with IL36RN gene mutations. Pediatric Dermatol. 2017;34(Supplement 2):S46.

21. Cordoro KM, Ucmak D, Hitraya-Low M, Rosenblum MD, Liao W. Response to interleukin (IL)-17 inhibition in an adolescent with severe manifestations of IL-36 receptor antagonist deficiency (DITRA). JAMA Dermatol. 2017;153:106-8.

22. Caorsi R, Minoia F, Viglizzo G, et al. The therapeutic challenge of DITRA syndrome. Pediatr Rheumatol. 2017;15(Supplement):1.

23. Glerup M, Veirum J, Iversen L, Christiansen M, Herlin T. Generalized pustular psoriasis in infant with heterozygous mutation in the IL36RN gene successfully treated with infliximab. Pediatr Rheumatol Online J. 2014;12(Suppl 1):P79.

24. Mendonca LO, Malle L, Donovan FX, et al. Deficiency of interleukin-1 receptor antagonist (DIRA): report of the first Indian patient and a novel deletion affecting IL1RN. J Clin Immunol. 2017;37: 445-51.

25. Panju AH, Bell CM. Policy alternatives for treatments for rare diseases. CMAJ. 2010;182(17): E787-92.

26. Gagnier JJ, Kienle G, Altman DG, Moher D, Sox H, Riley D, CARE Group. The CARE guidelines: consensus-based clinical case reporting guideline development. Glob Adv Health Med. 2013;2:38-43.

27. https://www.ncbi.nlm.nih.gov/clinvar?term=1476 79[MIM]. Accessed 12 Sep 2018.

28. https://www.ncbi.nlm.nih.gov/clinvar?term=6055 07[MIM]. Accessed 12 Sep 2018.

29. Minkis K, Aksentijevich I, Goldbach-Mansky R, et al. Interleukin 1 receptor antagonist deficiency presenting as infantile pustulosis mimicking infantile pustular psoriasis. Arch Dermatol. 2012;148:747-52.

30. Schnellbacher C, Ciocca G, Menendez R, et al. Deficiency of interleukin-1 receptor antagonist responsive to anakinra. Pediatr Dermatol. 2013;30:758-60.

31. Mendoní ALO, et al. A case report of a novel compound heterozygous mutation in a Brazilian patient with deficiency of IL1RA (DIRA). J Clin Immunol. 2017;37(Supplement 1):S19. 
32. Berdeli A, Sözeri B, Gerceker Türk B, Oz A, Mir S. Turkish DIRA patient with novel IL1RN gene mutation. Pediatr Rheumatol. 2015;13(Suppl 1):P181.

33. Ulusoy E, Karaca NE, El-Shanti H, Kilicoglu E, Aksu G, Kutukculer N. Interleukin-1 receptor antagonist deficiency with a novel mutation; late onset and successful treatment with canakinumab: a case report. J Med Case Rep. 2015;9:145.

34. Thacker PG, Binkovitz LA, Thomas KB. Deficiency of interleukin-1-receptor antagonist syndrome: a rare auto-inflammatory condition that mimics multiple classic radiographic findings. Pediatr Radiol. 2012;42:495-8.

35. Jesus AA, Osman M, Silva CA, et al. A novel mutation of IL1RN in the deficiency of interleukin-1 receptor antagonist syndrome: description of two unrelated cases from Brazil. Arthritis Rheum. 2011;63:4007-17.

36. Stenerson M, Dufendach K, Aksentijevich I, Brady J, Austin J, Reed AM. The first reported case of compound heterozygous IL1RN mutations causing deficiency of the interleukin-1 receptor antagonist. Arthritis Rheum. 2011;63:4018-22.

37. Hüffmeier $U$, Wätzold $M$, Mohr J, Schön MP, Mössner R. Successful therapy with anakinra in a patient with generalized pustular psoriasis carrying IL36RN mutations. Br J Dermatol. 2014;170:202-4.

38. Boehner A, Navarini AA, Eyerich K. Generalized pustular psoriasis-a model disease for specific targeted immunotherapy, systematic review. Exp Dermatol. 2018;27:1067-77.

39. Akiyama M, Takeichi T, McGrath JA, Sugiura K. Autoinflammatory keratinization diseases. J Allergy Clin Immunol. 2017;140:1545-7.

40. Akiyama M, Takeichi T, McGrath JA, Sugiura K. Autoinflammatory keratinization diseases: an emerging concept encompassing various inflammatory keratinization disorders of the skin. J Dermatol Sci. 2018;90:105-11. 